\title{
Unexpected Solvent Influence on the Rheology of
}

\section{Supramolecular Polymers}

Bruno G. Alvarenga ${ }^{l}$, Matthieu Raynal $^{2}$, Laurent Bouteiller $^{2} *$ and Edvaldo Sabadini $^{l} *$

${ }^{1}$ Department of Physical-Chemistry, Institute of Chemistry, University of Campinas UNICAMP, Brazil

sabadini@iqm.unicamp.br

${ }^{2}$ Sorbonne Universités, UPMC Univ Paris 06, CNRS, Institut Parisien de Chimie Moléculaire, Equipe Chimie des Polymères, 4 Place Jussieu, F-75005 Paris, France.

laurent.bouteiller@upmc.fr

ABSTRACT. A very limited change in the nature of the solvent (toluene versus pentylbenzene) can have an unexpectedly strong effect on the viscosity of a hydrogen bonded supramolecular polymer. In pentylbenzene, slightly stronger hydrogen bonds are formed than in toluene, but the supramolecular polymer solution is c.a. 25 times less viscous. Several hypotheses can be envisaged to explain this counterintuitive result. We propose that this effect is actually related to a solvation effect involving the outer corona of the supramolecular objects. When the cohesive energy density of the solvent approaches the cohesive energy density of the outer corona of the 
supramolecular objects, the viscosity of solution is reduced, possibly because of faster local dynamics.

\section{Introduction}

The choice of the solvent has a strong influence on the outcome of any self-assembly process, which makes the rationalization of solvent effects of paramount importance. In the case of outof-equilibrium processes such as crystal engineering, ${ }^{1-3}$ organogelation, ${ }^{4-6}$ or the processing of nanostructures, ${ }^{7}$ such rationalization is particularly challenging. At first glance, the case of systems that are at thermodynamic equilibrium seems to be much better understood. For instance, in the case of hydrogen bonded assemblies, the main solvent characteristic is known to be its polarity. The influence of the hydrogen bond competition by the solvent polar groups can even be quantitatively accounted for ${ }^{8,9}$ Still, when considering solvents of similar polarity, subtle effects can occur that significantly alter the outcome of the assembly structure and properties. For example, the bulkiness of aromatic solvent molecules has been shown to control the stability of tubular supramolecular assemblies because of the necessity for the solvent to fit inside the cavities. ${ }^{10}$ Similarly, replacing a cyclic alkane solvent by a linear alkane has been shown to change the inner conformation or even the helical sense of self-assembled rods, ${ }^{11-13}$ possibly because the thinner linear alkanes are able to intercalate between the alkyl chains of the stacked monomers. Even the length of alkane molecules has been shown to influence the stability of stacked hydrogen bonded dimers through a strong odd-even effect. ${ }^{14}$

A better understanding of these and possibly other subtle solvent effects is clearly required before more complex issues such as the influence of solvent on crystal growth can be envisaged. Supramolecular polymers, i.e. long chains of non-covalently and reversibly linked repeat units, are ideal tools to study such effects because their large size can reveal cooperative growth ${ }^{15}$ or 
chiral amplification effects ${ }^{16}$ that can conveniently be probed by spectroscopic tools and that sometimes even result in macroscopic property changes. Moreover, the absence of chain bundling avoids the complications associated with kinetic traps that can occur during the growth of other large size systems such as crystalline fibers.

The observation that a particular hydrogen bonded supramolecular polymer (see below) forms more viscous solutions in toluene than in dodecane (see Figure 1) although dodecane is significantly less polar triggered our interest. We report here a careful study of this surprising effect and show that within the family of alkylbenzene solvents, an increase in the alkyl chain length leads to stronger hydrogen bonds but also to much less viscous solutions. We discuss possible interpretations for this highly counter-intuitive effect.

\section{Experimental Section}

Bis-urea EHUT was synthetized as previously described. ${ }^{17}$ All aliphatic and aromatic solvents were obtained from Sigma-Aldrich and used without purification. The amount of water $(<200$ ppm) in the solvents was determined by Karl-Fisher analysis.

Sample preparation. The solutions of EHUT were prepared at $80^{\circ} \mathrm{C}$ in a sand bath, under stirring at least 1 day. Then, the temperature was decreased at $5^{\circ} \mathrm{C} /$ hour to room temperature.

Rheological measurements. Flow curves and oscillatory measurement of $6 \mathrm{mmol} \mathrm{L}^{-1}$ and $10 \mathrm{mmol} \mathrm{L}^{-1}$ EHUT solutions were obtained with a Haake RheoStress 1 rheometer equipped with a Z20-din cell (volume: $8.2 \mathrm{~mL}$; gap: $4.2 \mathrm{~mm}$ ). The temperature was controlled at $20.0^{\circ} \mathrm{C}$ by an external water-bath system with precision better than $0.1{ }^{\circ} \mathrm{C}$. The flow curves were obtained over a shear rate from $10^{-4}$ to $1.0 \mathrm{~s}^{-1}$. The oscillatory experiments were carried out within linear viscoelastic range as determined through strain sweep measurements. A solvent-trap accessory 
was used to minimize solvent evaporation. All experiments were carried out at least in duplicates.

Infrared Spectroscopy. Spectra for $1 \mathrm{mmol} \mathrm{L}^{-1}$ EHUT solutions were measured on a Nicolet iS10 spectrometer using a $\mathrm{CaF}_{2}$ cell with $1 \mathrm{~mm}$ pathlength. The spectra were corrected for air, solvent and cell absorption. The temperature was controlled with a heating device from Specac (P/N21525). Consecutive spectra were obtained separated by at least $15 \mathrm{~min}$ to allow thermal equilibration. Thermal expansion of solutions was not corrected.

Differential Scanning Calorimetry. Thermograms were measured using an N-DSCIII instrument from CSC. The reference cell was filled with solvent and the sample cell $(0.3 \mathrm{~mL})$ with $10 \mathrm{mmol} \mathrm{L}{ }^{-1}$ EHUT solution, and a constant pressure of $510^{5} \mathrm{~Pa}$ was applied. A baseline scan (solvent in both reference and sample cells) was performed in identical conditions and subtracted from the sample scan. Transition temperature $\left(\mathrm{T}^{* *}\right)$ was taken as the average of heating and cooling scans, at a scan rate of $1{ }^{\circ} \mathrm{C} \mathrm{min}^{-1}$.

Isothermal Titration Calorimetry. The EHUT heats of dissociation were measured using a MicroCal VP-ITC titration microcalorimeter. Aliquots $(5 \mu \mathrm{L})$ of $0.25 \mathrm{mmol} \mathrm{L}^{-1}$ EHUT solutions in a syringe were automatically injected into a continuously stirred (310 rpm) isothermal cell $\left(1.420 \mathrm{~cm}^{3}\right)$ containing the pure solvent.

\section{Results and Discussion}

Bis-urea EHUT (Figure 1) is known to self-assemble into a long hydrogen bonded tubular structure in low polarity solvents. ${ }^{18,19}$ In the semi-dilute regime, the entanglements between these long and rigid objects (that can reversibly break and recombine) yield viscoelastic solutions. While the rheological properties of these solutions have been studied in several aliphatic or 
aromatic solvents,$^{20}$ the results in the various solvents have never been quantitatively compared. Because the dielectric constant is lower in dodecane than in toluene, one expects hydrogen bonded assemblies to be stronger in dodecane. Therefore it is surprising that the EHUT solution seems to be less viscous in dodecane than in toluene (based on the qualitative comparison of the solutions, Figure 1). In order to investigate this effect, we systematically characterized the rheology and the structure of this supramolecular polymer in a range of aliphatic and aromatic solvents.

(a)

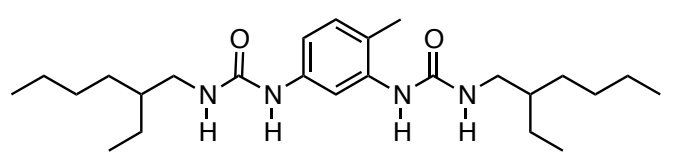

(b)

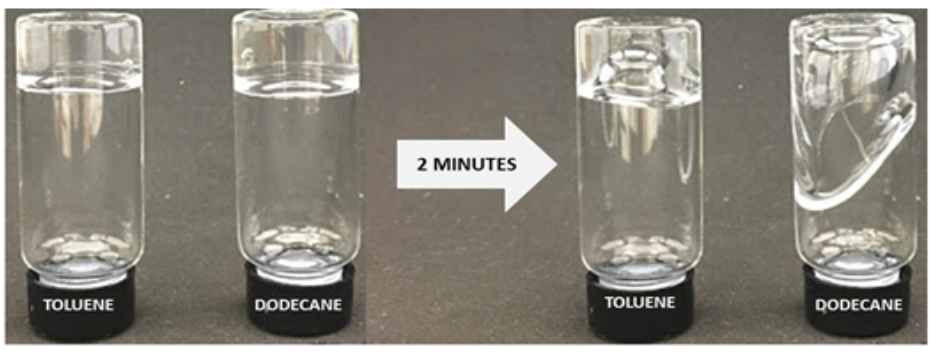

Figure 1. Structure of EHUT (a) and photographs of EHUT solutions in toluene or n-dodecane (b) 10 seconds (left) and 2 minutes (right) after inverting the vials $\left(20 \mathrm{mmol} \mathrm{L}^{-1}, 20{ }^{\circ} \mathrm{C}\right)$.

Figure 2 shows the zero shear viscosity (obtained at the Newtonian plateau) of EHUT solutions in alkylbenzenes (toluene to pentylbenzene) and in linear alkanes (heptane to dodecane), that were obtained from the flow curves (Figures S1 and S2). In order to isolate the contribution of the solute from the solvent bulk viscosity, the zero shear viscosity of the solution (Figure 2) was normalized by the viscosity of the pure solvents (Figure S3). First of all, this data confirms the visual observation of Figure 1: the toluene solution is approximately five times more viscous than the dodecane one (Figure S3). Moreover, different trends are detected in 
aromatic and aliphatic solvents when the length of the solvent molecule is increased. The relative viscosity is roughly constant within the aliphatic series, which is expected because of the weak effect of the alkane size on the solvent properties. In particular, the polarity of the aliphatic solvents is very similar in heptane and dodecane, which means that the strength of the hydrogen bonds formed between the EHUT molecules is expected to be similar. In contrast, the relative viscosity of the solutions strongly decreases within the aromatic series: the relative viscosity is c.a. 25 times lower in pentylbenzene than in toluene although the former solvent is the least polar of the series. In the following we therefore focus our attention on the aromatic solvents.

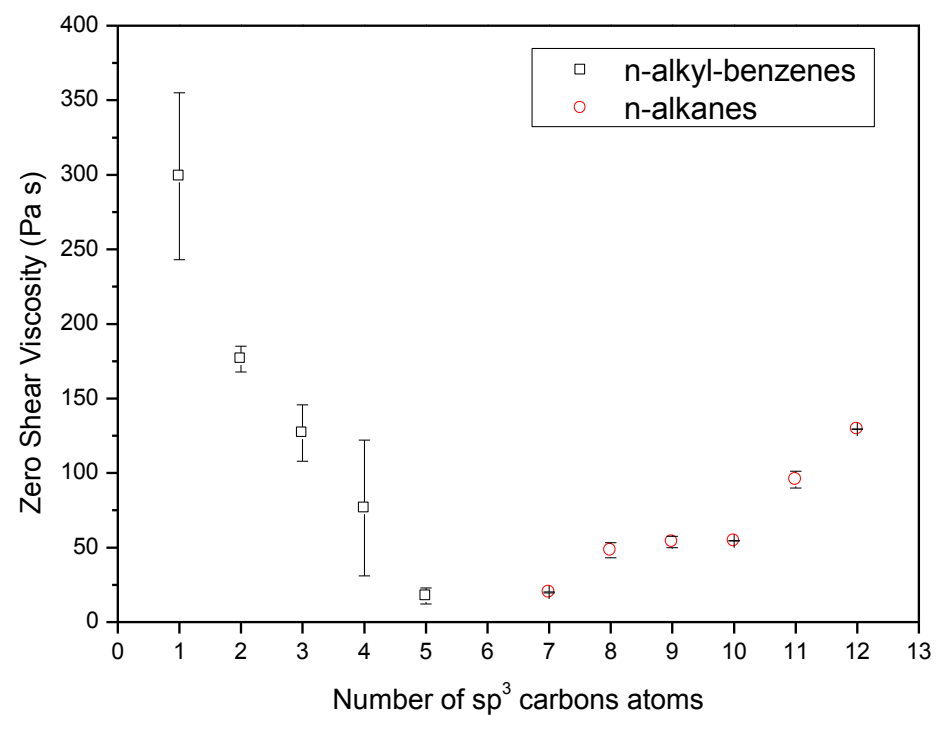

Figure 2. Dependence of the zero shear viscosity for EHUT solutions $\left(10 \mathrm{mM}, 20{ }^{\circ} \mathrm{C}\right)$ in aromatic (squares) or aliphatic (circles) solvents versus the number of $\mathrm{sp}^{3}$ carbon atoms in the solvent (1 for toluene; 12 for dodecane).

The previous viscosity measurements were complemented by oscillatory experiments in order to probe the dynamics of the supramolecular polymer. The variation of the elastic $\left(\mathrm{G}^{\prime}\right)$ and viscous (G”) moduli as a function of the frequency for solutions in toluene or pentylbenzene is 
shown in Figure 3 (the curves for the other solvents can be seen in Figure S4). The elastic and viscous moduli can be analyzed by considering the Maxwell model (Equations 1 and 2).

$$
\begin{aligned}
& G^{\prime}(\omega)=\frac{\omega^{2} \tau_{R}^{2}}{1+\omega^{2} \tau_{R}^{2}} G_{0} \\
& G^{\prime \prime}(\omega)=\frac{\omega \tau_{R}}{1+\omega^{2} \tau_{R}^{2}} G_{0}
\end{aligned}
$$

Although the Maxwell model does not fit perfectly the curves, it can be used to discuss the results. According to this model, the dynamics of the system can be described by a single relaxation time $\tau_{R}$, obtained from the cross-over frequency of the two moduli $\left(\omega_{c}=1 / \tau_{R}\right.$ when $\left.\mathrm{G}^{\prime}\left(\omega_{\mathrm{c}}\right)=\mathrm{G}^{\prime \prime}\left(\omega_{\mathrm{c}}\right)\right){ }^{19,21}$ The relaxation time measured in toluene is c.a. 20 times longer than in pentylbenzene, although the plateau moduli are very similar. Therefore, we propose that the decrease in viscosity from toluene to pentylbenzene is actually associated with the relaxation times, more specifically with an increase in the dynamics in pentylbenzene.

For chains with a reversible backbone, the shear stress can relax by two mechanisms: reptation and scission of the chains, that are described by two characteristic relaxation times, the reptation time, $\tau_{\text {rep }}$ and the breaking time, $\tau_{b}$. If the scission is much faster than the reptation $\left(\tau_{\text {rep }} \gg>\tau_{b}\right)$, the rheological behavior is perfectly described by the Maxwell Model, with a single exponential decay with a relaxation time, $\tau_{\mathrm{R}}$, given by the following expression: ${ }^{22}$

$$
\tau_{R}=\sqrt{\tau_{r e p} \tau_{b}}
$$

Such phenomenon was well described for gel-like solutions of wormlike micelles formed by combination of a cationic surfactant with hydrotropes such as salicylate. ${ }^{23}$

The high viscosity of EHUT supramolecular polymer in toluene and the associated large value of $\tau_{R}$ can therefore be due to a longer breaking time or to a longer reptation time (or both) in 
toluene than in pentylbenzene. A priori, this evolution of the relaxation times (and the viscosity) induced by the solvent could be due to a variety of reasons that we now examine.
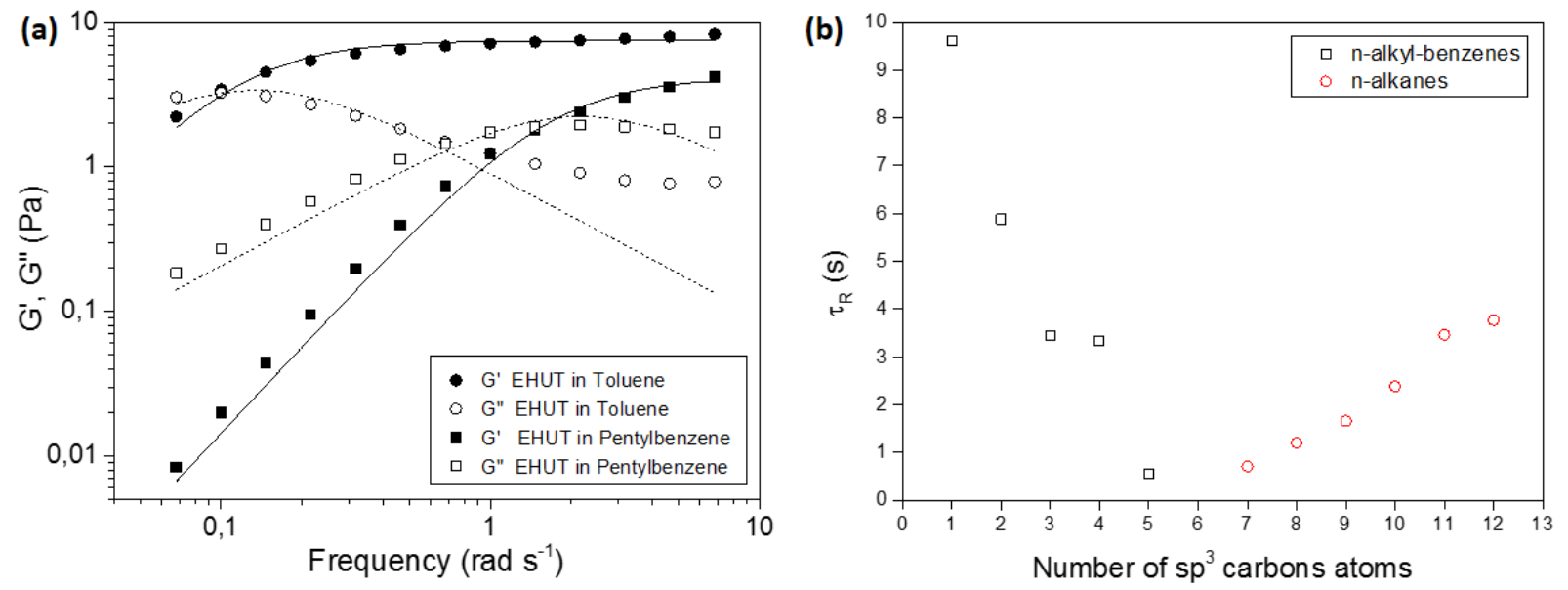

Figure 3. (a) Dynamic moduli versus oscillation frequency for EHUT in toluene or pentylbenzene $\left(6 \mathrm{mmol} \mathrm{L}{ }^{-1}, 20^{\circ} \mathrm{C}\right)$. The lines correspond to fits with the Maxwell model. (b) Dependence of the cross-over frequency for EHUT solutions in aromatic or aliphatic solvents versus the number of $\mathrm{sp}^{3}$ carbon atoms in the solvent.

First of all, the water content in the solvent is known to affect the viscosity of hydrogen bonded supramolecular polymers. ${ }^{24}$ Therefore we checked by Karl-Fischer analysis that all the solvents we used had approximately the same water content (from 80 to $200 \mathrm{ppm}$ ). We can therefore exclude that the decrease in viscosity in the aromatic series is due to different water contents.

We also need to check if the structure of the supramolecular polymer is the same in the various aromatic solvents. Indeed, previous studies in toluene have shown that the tubular structure is the main supramolecular structure present at $10 \mathrm{mM}$ and $20^{\circ} \mathrm{C}$, but that the supramolecular tubes convert into thinner filaments at higher temperatures. ${ }^{25}$ Since the viscosity of the filaments is 
lower than the one of the tubes, we have to check whether the unexpected viscosity trend is related to a possible influence of the solvent on the tube to filament equilibrium. ${ }^{10}$ The shape of the N-H vibration band measured by FTIR spectroscopy has been shown to be characteristic of the supramolecular structure of EHUT ${ }^{18}$ Figure 4 shows that the FTIR spectra in toluene and in pentylbenzene are identical and that their shape is characteristic of the tube structure. Then, the thermal stability of tube supramolecular assembly was probed both by FTIR and by DSC experiments (Figure 5). The data show that the transition temperature between tubes and filaments actually increases from toluene to pentylbenzene. The study of the phase diagram of EHUT in toluene has shown that the filament concentration is negligible at $20^{\circ} \mathrm{C}$ for $[\mathrm{EHUT}]=$

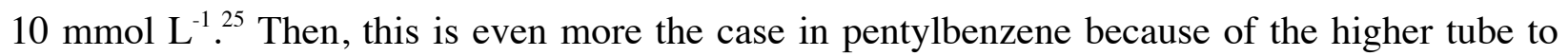
filament transition temperature in this solvent. Therefore, it is safe to consider that the tube supramolecular structure is the only type of assembly present at the temperature and concentrations of the rheological experiments of Figure 2 and 3; i.e. the evolution of viscosity in the aromatic solvents is not due to a change in supramolecular structure. ${ }^{26}$

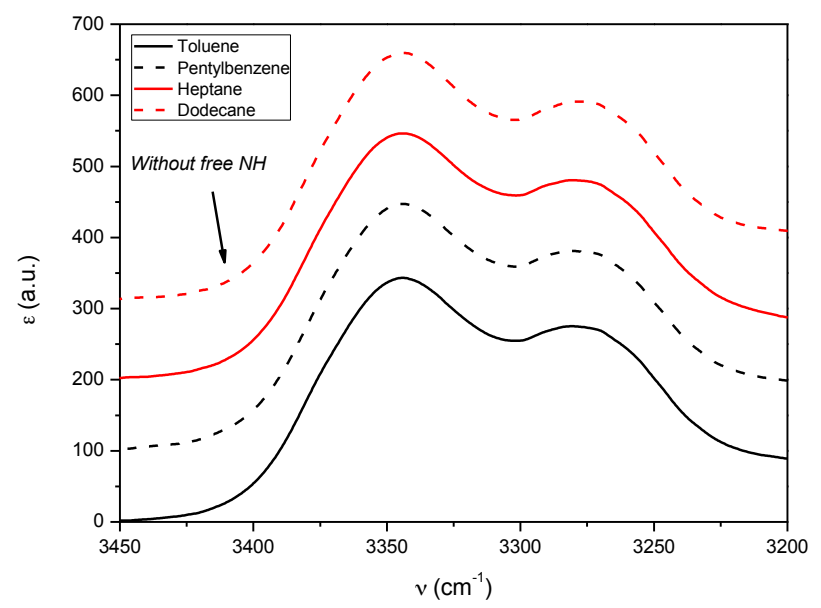


Figure 4. FTIR spectra for EHUT solutions in aromatic or aliphatic solvents $\left(10 \mathrm{mmol} \mathrm{L}^{-1}, 20\right.$ $\left.{ }^{\circ} \mathrm{C}\right)$.
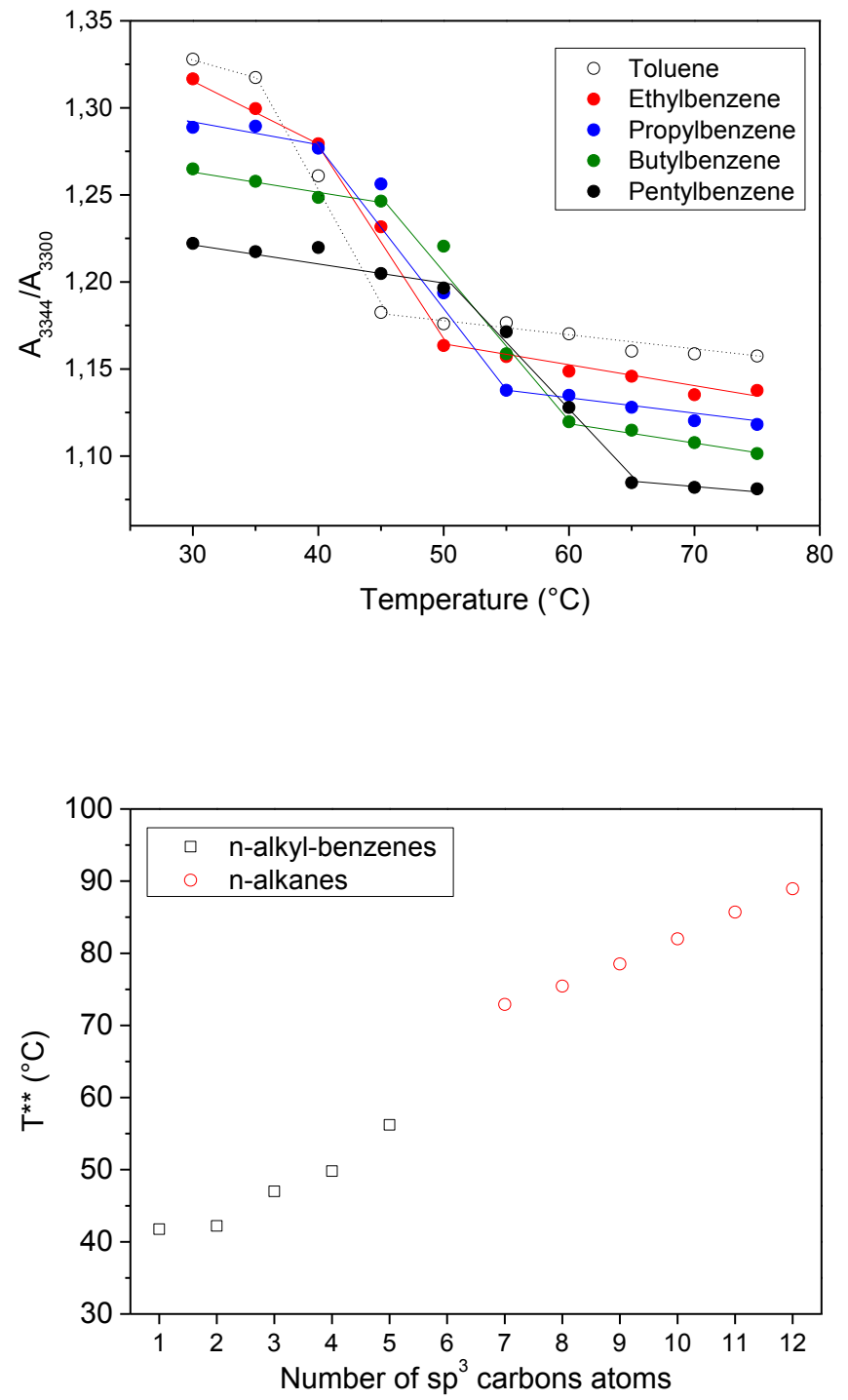

Figure 5. Transition between tube and filament structure for EHUT solutions in aromatic solvents, measured by (a) the shape of the FTIR band $\left(1 \mathrm{mmol} \mathrm{L}^{-1}\right)$, or (b) DSC $\left(10 \mathrm{mmol} \mathrm{L}^{-1}\right)$.

In order to see if a change in length of the assemblies could be a relevant explanation, FTIR spectroscopy was used to qualitatively probe the strength of hydrogen bonds in these solvents. 
Figure 4 shows that EHUT is fully hydrogen bonded in both solvents (at $10 \mathrm{mM}$ and $20^{\circ} \mathrm{C}$ ), i.e. no free N-H vibration band is detected. In order to favor dissociation of the assemblies, the concentration was reduced to $1 \mathrm{mM}$ and the temperature was increased. Figure 6 shows that at $75^{\circ} \mathrm{C}$, a small contribution of free $\mathrm{N}-\mathrm{H}$ groups can be detected in toluene, but not in pentylbenzene. Although at this temperature, the main assembly in both solvents is not the tube (but the filament), this experiment confirms the expectation that the hydrogen bonds are stronger in pentylbenzene than in toluene because of the lower dielectric constant of the former solvent (see Figure S5).
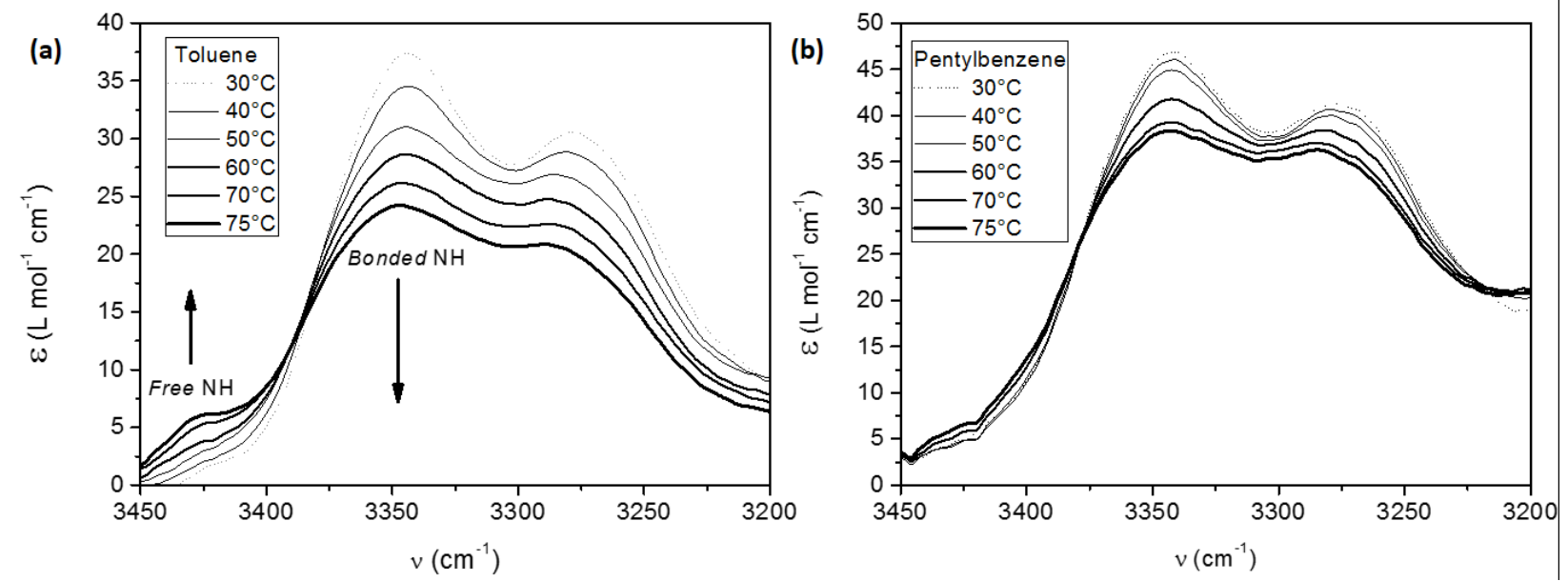

Figure 6. FTIR spectra for EHUT solutions in toluene (a) or pentylbenzene (b) $\left(1 \mathrm{mmol} \mathrm{L}^{-1}\right)$.

In an attempt to obtain more quantitative information about the self-assembly process, isothermal titration calorimetry (ITC) was used. Figure 7 shows the enthalpograms obtained when a $0.25 \mathrm{mmol} \mathrm{L} \mathrm{L}^{-1}$ EHUT solution is injected into the corresponding pure solvent at $25^{\circ} \mathrm{C}$. The endothermal heat effects detected are proportional to the amount of hydrogen bonds broken during the dilution. The fact that the heat effects are smaller and shifted to lower concentrations in the case of pentylbenzene means that the assemblies are stronger (i.e. more difficult to break) in this solvent. ${ }^{27}$ Unfortunately, the data did not allow a consistent fit that would have yielded the 
thermodynamic parameters for the supramolecular polymerization in pentylbenzene. Still the ITC and FTIR data both indicate qualitatively a stronger hydrogen bonding in pentylbenzene than in toluene, which probably means that the supramolecular polymers are longer in pentylbenzene. ${ }^{28}$ Therefore, the solvent effect on the length of the assemblies cannot explain the lower viscosity in pentylbenzene.

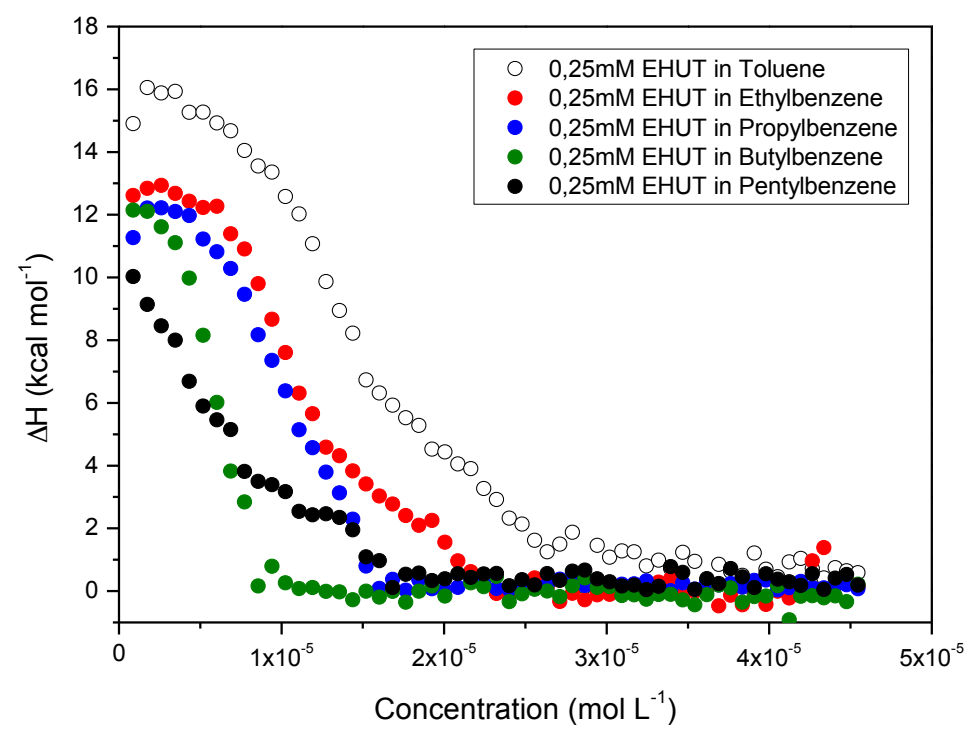

Figure 7. ITC enthalpograms for $0.25 \mathrm{mmol} \mathrm{L}^{-1}$ EHUT solutions in aromatic solvents injected into the same pure solvent $\left(25^{\circ} \mathrm{C}\right)$.

If the faster macroscopic dynamics measured by rheology in pentylbenzene is not due to shorter chains, it has to be due to faster local dynamics; i.e. to faster scission and recombination of the tubes in pentylbenzene than in toluene. The independent influence of dynamics and thermodynamics has actually been unambiguously exemplified by S. L. Craig et al. with metalligand systems. ${ }^{29}$ In our case, the reason why the hydrogen bonded tubes (that are more stable in pentylbenzene) would nevertheless break and recombine faster than in toluene, is not 
immediately obvious. However, the reason may be related to a subtle solvation effect. Indeed, the cohesive energy density of the solvent, measured by the Hildebrand solubility parameter, decreases significantly from $18.2 \mathrm{MPa}^{1 / 2}$ for toluene to $17.4 \mathrm{MPa}^{1 / 2}$ for pentylbenzene. ${ }^{30}$ Therefore, the 2-ethylhexyl chains of EHUT $\left(16 \mathrm{MPa}^{1 / 2}\right)$ that form a corona around the nanotube are better solvated in pentylbenzene than they are in toluene. Moreover, the scission of a tube in two parts involves the formation of two new ends that necessitate new solvation shells. We propose that the creation of these additional solvation shells is a faster process in a solvent (pentylbenzene) that better solvates the 2-ethylhexyl corona.

Actually, another possible consequence of the better solvation of the 2-ethylhexyl corona in pentylbenzene is that tube-tube contacts that occur at entanglement points could relax faster than in toluene, therefore accelerating the dynamics of tube-tube interactions. At this point, we cannot discriminate between an intra-tube effect (i.e. faster scission and recombination) or an inter-tube effect (i.e. faster disentanglement), but the parallel evolution of the overall dynamics and of the cohesive energy density point toward the strong influence of solvation.

\section{Conclusion}

In conclusion, we report that a very limited change in the nature of the solvent can have an unexpectedly strong effect on the viscosity of a hydrogen bonded supramolecular polymer. To our best knowledge, this is the first example where slightly stronger hydrogen bonding actually leads to much less viscous solutions. We propose that this effect is actually related to a solvation effect involving the outer corona of the supramolecular objects. When the cohesive energy density of the solvent approaches the cohesive energy density of the outer corona of the supramolecular objects, the viscosity of solution is reduced, possibly because of faster local 
dynamics. Further work is needed to assess the possibility of tuning the dynamics of a supramolecular polymer by changing the nature of the solvent.

Supporting Information. Additional rheology data. This material is available free of charge via the Internet at http://pubs.acs.org/.

\section{Acknowledgements}

The authors thank the Conselho Nacional de Desenvolvimento Científico e Tecnológico (CNPq, Brasília, Brazil) and the Fundação de Amparo à Pesquisa do Estado de São Paulo (FAPESP, São Paulo, Brazil) for financial support and fellowships.

\section{References}

(1) Goesten, M. G.; Kapteijn, F.; Gascon, J. Fascinating Chemistry or Frustrating Unpredictability: Observations in Crystal Engineering of Metal-Organic Frameworks. CrystEngComm 2013, 15, 9249-9257.

(2) Blagden, N.; Berry, D. J.; Parkin, A.; Javed, H.; Ibrahim, A.; Gavan, P. T.; De Matos, L. L.; Seaton, C. C. Current Directions in Co-Crystal Growth. New J. Chem. 2008, 32, 1659-1672.

(3) Lovette, M. A.; Robben Browning, A.; Griffin, D. W.; Sizemore, J. P.; Snyder, R. C.; Doherty, M. F. Crystal Shape Engineering. Ind. Eng. Chem. Res. 2008, 47, 9812-9833.

(4) Lan, Y.; Corradini, M. G.; Weiss, R. G.; Raghavan, S. R.; Rogers, M. A. To Gel or Not to Gel: Correlating Molecular Gelation with Solvent Parameters. Chem. Soc. Rev. 2015, 44, 60356058 .

(5) Bonnet, J.; Suissa, G.; Raynal, M.; Bouteiller, L. Organogel Formation Rationalized by Hansen Solubility Parameters: Dos and Don'ts. Soft Matter 2014, 10, 3154-3160.

(6) Dasgupta, D.; Guenet, J.-M. The Solvent in Physical Gelation: Polymers Versus Organogelators. Macromol. Chem. Phys. 2013, 214, 1885-1892.

(7) Chen, Y.; Zhan, C.; Yao, J. Understanding Solvent Manipulation of Morphology in BulkHeterojunction Organic Solar Cells. Chem. - Asian J. 2016, 11, 2620-2632.

(8) Cook, J. L.; Hunter, C. A.; Low, C. M. R.; Perez-Velasco, A.; Vinter, J. G. Solvent Effects on Hydrogen Bonding. Angew. Chem. Int. Ed. 2007, 46, 3706-3709.

(9) Amenta, V.; Cook, J. L.; Hunter, C. A.; Low, C. M. R.; Sun, H.; Vinter, J. G. Interplay of Self-Association and Solvation in Polar Liquids. J. Am. Chem. Soc. 2013, 135, 12091-12100. 
(10) Pinault, T.; Isare, B.; Bouteiller, L. Solvents with Similar Bulk Properties Induce Distinct Supramolecular Architectures. ChemPhysChem 2006, 7, 816-819.

(11) Cantekin, S.; Nakano, Y.; Everts, J. C.; van der Schoot, P.; Meijer, E. W.; Palmans, A. R. A. A Stereoselectively Deuterated Supramolecular Motif to Probe the Role of Solvent during Self-Assembly Processes. Chem. Commun. 2012, 48, 3803-3805.

(12) Nakano, Y.; Hirose, T.; Stals, P. J. M.; Meijer, E. W.; Palmans, A. R. A. Conformational Analysis of Supramolecular Polymerization Processes of Disc-like Molecules. Chem. Sci. 2012, $3,148-155$.

(13) Nakano, Y.; Markvoort, A. J.; Cantekin, S.; Filot, I. A. W.; ten Eikelder, H. M. M.; Meijer, E. W.; Palmans, A. R. A. Conformational Analysis of Chiral Supramolecular Aggregates: Modeling the Subtle Difference between Hydrogen and Deuterium. J. Am. Chem. Soc. 2013, 135, 16497-16506.

(14) Jonkheijm, P.; van der Schoot, P.; Schenning, A. P. H. J.; Meijer, E. W. Probing the Solvent-Assisted Nucleation Pathway in Chemical Self-Assembly. Science 2006, 313, 80-83.

(15) De Greef, T. F. A.; Smulders, M. M. J.; Wolffs, M.; Schenning, A. P. H. J.; Sijbesma, R. P.; Meijer, E. W. Supramolecular Polymerization. Chem. Rev. 2009, 109, 5687-5754.

(16) Palmans, A. R. A.; Meijer, E. W. Amplification of Chirality in Dynamic Supramolecular Aggregates. Angew. Chem. Int. Ed. 2007, 46, 8948-8968.

(17) Lortie, F.; Boileau, S.; Bouteiller, L.; Chassenieux, C.; Deme, B.; Ducouret, G.; Jalabert, M.; Laupretre, F.; Terech, P. Structural and Rheological Study of a Bis-Urea Based Reversible Polymer in an Apolar Solvent. Langmuir 2002, 18, 7218-7222.

(18) Bouteiller, L.; Colombani, O.; Lortie, F.; Terech, P. Thickness Transition of a Rigid Supramolecular Polymer. J. Am. Chem. Soc. 2005, 127, 8893-8898.

(19) Shikata, T.; Nishida, T.; Isare, B.; Linares, M.; Lazzaroni, R.; Bouteiller, L. Structure and Dynamics of a Bisurea-Based Supramolecular Polymer in N-Dodecane. J. Phys. Chem. B 2008, $112,8459-8465$.

(20) Isare, B.; Pensec, S.; Raynal, M.; Bouteiller, L. Bisurea-Based Supramolecular Polymers: From Structure to Properties. Comptes Rendus Chim. 2016, 19, 148-156.

(21) Francisco, K. R.; Dreiss, C. A.; Bouteiller, L.; Sabadini, E. Tuning the Viscoelastic Properties of Bis(urea)-Based Supramolecular Polymer Solutions by Adding Cosolutes. Langmuir 2012, 28, 14531-14539.

(22) Cates, M. E. Reptation of Living Polymers: Dynamics of Entangled Polymers in the Presence of Reversible Chain-Scission Reactions. Macromolecules 1987, 20, 2289-2296.

(23) Rehage, H.; Hoffmann, H. Rheological Properties of Viscoelastic Surfactant Systems. $J$. Phys. Chem. 1988, 92, 4712-4719.

(24) Louhichi, A.; Jacob, A. R.; Bouteiller, L.; Vlassopoulos, D. Effect of Humidity on 
Viscoelastic Properties of Living Supramolecular Polymers. to be published.

(25) Bellot, M.; Bouteiller, L. Thermodynamic Description of Bis-Urea Self-Assembly: Competition between Two Supramolecular Polymers. Langmuir 2008, 24, 14176-14182.

(26) The fact that the transition enthalpies are similar in all the aromatic solvents (ca. $1 \mathrm{kcal} / \mathrm{Mol}$ ) is also consistent with the formation of the same supramolecular structure.

(27) Arnaud, A.; Bouteiller, L. Isothermal Titration Calorimetry of Supramolecular Polymers. Langmuir 2004, 20, 6858-6863.

(28) In the case of an isodesmic growth, stronger binding means a larger association constant, which implies longer chains. However, in the case of a cooperative growth, it is mathematically possible that larger association constants actually leads to shorter chains if the cooperativity is weaker. Unfortunately, the ITC data in pentylbenzene does not allow a quantitative fit of the data that could settle this point.

(29) Yount, W. C.; Loveless, D. M.; Craig, S. L. Small-Molecule Dynamics and Mechanisms Underlying the Macroscopic Mechanical Properties of Coordinatively Cross-Linked Polymer Networks. J. Am. Chem. Soc. 2005, 127, 14488-14496.

(30) Hansen, C. M.; Editor. Hansen Solubility Parameters: A User's Handbook.; CRC Press LLC, 2007. 
for Table of Contents use only

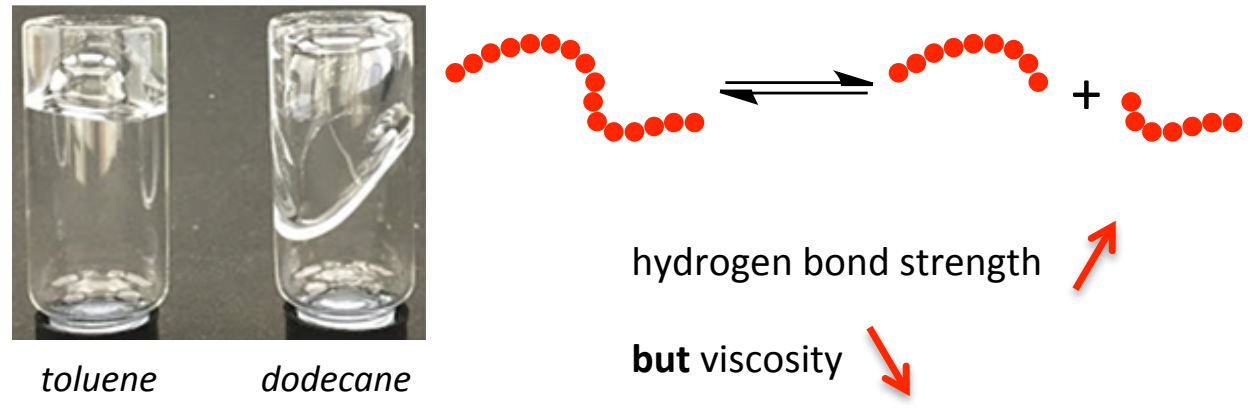

